\title{
Modulation of wave-current interactions by horizontal mixing and spatial resolution
}

\author{
Bennis A.-C. ${ }^{1,{ }^{*}}$, Dumas Franck ${ }^{2}$, Blanke Bruno $^{3}$
}

${ }^{1}$ Laboratoire de Morphodynamique Continentale et Côtière, UMR 6143, CNRS-UNICAEN-UR, 24 rue

des Tilleuls, Caen 14000, France

${ }^{2}$ Dyneco/Physed, Ifremer Centre de Bretagne, ZI Pointe du diable, Plouzané 29280, France

${ }^{3}$ Laboratoire de Physique des Océans, UMR 6523, CNRS-Ifremer-UBO-IRD, 6 Avenue Le Gorgeu, 29238 Brest cedex 3, France

* Corresponding author : Anne-Claire Bennis, Tel.: +33231565718; fax: +33231565757 ;

email address : anne-claire.bennis@unicaen.fr

\begin{abstract}
:
The mechanics of rip currents are complex, involving interactions between waves, currents, water levels and bathymetry that pose particular challenges for numerical modeling. Horizontal turbulent diffusion in a rip system is difficult to measure using dye dilution or surfzone drifters, as shown by the range of published values for the horizontal diffusion coefficient. Here, we studied the effects of horizontal mixing on wave-current interactions by testing several diffusivity estimates in a fully coupled 3D wave-current model run at two different spatial resolutions. Published results using very low diffusion have found that near the shore the wave rays converge towards the rip channel because of refraction by the currents. We showed that this process is modulated by both horizontal mixing and spatial resolution. We found that, without the feedback of currents on waves, the flow is more sensitive to horizontal mixing, with large alterations, especially offshore, and generally lower velocities. These modifications ascribed to mixing are similar to those induced by the feedback mechanism. When a large mixing coefficient is used: (i) the behavior of the rip system is similar for both coupling modes (i.e., with and without the feedback of currents on waves) and for each resolution; and (ii) the evolution of the flow is more stable over time. Lastly, we show that the horizontal mixing strongly decreases the intensity of the 3D rip velocity, but not its vertical shear, which is mainly dependent on the vertical mixing scheme and on the forcing terms.
\end{abstract}

\section{Highlights}

- We continue the 2D work of Weir et al. (2011) with 3D simulations. Reference results depend on both horizontal mixing and spatial resolution. CEW and horizontal mixing act on the flow in the same way. We express wave-current interactions in term of horizontal mixing. The vertical shear of the rip velocity is not changed by horizontal mixing and CEW.

Keywords : Coastal ocean modelling, Nearshore processes, Rip currents, Wave-current interactions, Horizontal mixing 


\section{Introduction}

Rip currents were first investigated by Shepard (1936), and are studied for their important influence on nearshore morphodynamics, cross-shore exchanges, and the hazard they pose to swimmers (MacMahan et al., 2006). From observations made on La Jolla beach in California, Shepard et al. (1941) described the rip structure as being composed of three major features: the feeder, the neck and the head. Scientific studies began as qualitative descriptions based on observations. Since then, theoretical and numerical approaches have been developed for rip currents, using observations for validation.

Rip currents are generated by the hydrodynamic instability resulting from wave refraction by currents (Leblond and Tang, 1974; Falques et al., 1999; Yu, 2006) and the convergence of longshore currents in regions of strong alongshore variations in wave conditions (Long and Ozkan-Haller, 2005). Transient currents forced by finite crest-length wave breaking also create a type of rip current (e.g., Johnson and Pattiaratchi, 2006; Spydell and Feddersen, 2009; Clark et al., 2012). The feedback mechanism between currents and waves has been reported by several authors (e.g., MacMahan et al., 2006) as playing an essential role in the development of the rip system. The main numerical studies considering the effects of currents on waves (hereafter CEW) reported the following behaviors: a) Leblond and Tang (1974) observed a modification of the flux of wave energy by the currents, with variability along the longshore coordinate; b) Haas et al. (1998) found that CEW reduce the offshore extent of rip currents; c) Yu and Slinn (2003) showed that rips are compacted with CEW because of the large decrease in the wave flux of momentum induced by wave breaking and explained that this decrease is due to changes in wave number (wave ray bending); and d) Weir et al. (2011) who complemented the last of these studies by calculating the change in the wavefield resulting from wave ray bending and from wave energy advected by the current (current flux of wave energy). The latter authors expressed wave-current interactions in terms of bottom friction.

Previous studies investigating the effects of current on waves were carried out without explicit turbulence modeling and at high resolution, namely 2 and 3 meters in both horizontal directions for Weir et al. (2011) and Yu and Slinn (2003), respectively. Because the systematic use of a spatial resolution lower than the size of the tiniest vortex is not possible, due to computational limitations, the effects of subgrid-scale processes must be parameterized in ocean models (e.g., Sagaut et al., 2013). This is particularly the case for horizontal diffusive mixing, which can be included in the horizontal 
momentum equations in several ways: i) modelers often use a diffusion coefficient that is a function of the grid size, but also of the flow and wave field characteristics (e.g., Smagorinsky, 1963; Okubo, 1971; Battjes, 1975); and ii) the discretization of the advection terms in an Eulerian framework with sigma-coordinates and the discretization of the Laplacian operator also generate numerical diffusion (Huang and Spaulding, 2002; Zwillinger, 1989).

Many studies have discussed this numerical diffusion (e.g., Fortunato and Baptista, 1994; Huang and Spaulding, 1996, 2002). Huang and Spaulding (2002) compared the advection scheme of Mellor and Blumberg (1985) with that of Fortunato and Baptista (1994). They found that both schemes produce large artificial diffusion, particularly where the bathymetry is steep. The most popular ocean models still use different versions of the schemes of Mellor and Blumberg (1985) (e.g., POM, ROMS or MARS) and of Fortunato and Baptista (1994) (e.g., POLCOMS).

The diffusion coefficient for nearshore applications is usually measured by dye dilution. The literature usually distinguishes between the absolute diffusivity, which characterizes the ensemble behavior of a cloud of particles, and the relative diffusivity related to the spreading of the cloud around its center of mass. Field studies provide many different values for the diffusion coefficient: i) Imann et al. (1971) found a cross-shore absolute diffusivity (hereafter $K_{a x}$ ) between $0.1 \mathrm{~m}^{2} \cdot \mathrm{s}^{-1}$ and $5.9 \mathrm{~m}^{2} . \mathrm{s}^{-1}$ and an alongshore absolute diffusivity (hereafter $K_{a y}$ ) between $0 \mathrm{~m}^{2} \cdot \mathrm{s}^{-1}$ and $0.17 \mathrm{~m}^{2} \cdot \mathrm{s}^{-1}$; ii) The longterm observations $\left(\mathcal{O}\left(10^{3}\right) s\right)$ of Grant et al. (2005) led to $K_{a y}$ between $40 \mathrm{~m}^{2} \cdot \mathrm{s}^{-1}$ and $80 \mathrm{~m}^{2} \cdot \mathrm{s}^{-1}$ with larger values for larger waves; and, iii) Clarke et al. (2007) noted that the absolute diffusivity was strongly dependent on wave conditions and on the presence of rip currents. Their assessment was in the range of $10^{-1} \mathrm{~m}^{2} \cdot \mathrm{s}^{-1}$ to $10^{3} \mathrm{~m}^{2} \cdot \mathrm{s}^{-1}$.

Brown et al. (2009) tested another measurement method in order to estimate diffusivity with more accuracy. They used thirty surfzone drifters at Sand City (Monterey Bay, California) over 7 days between April and May 2007. The beach is known for its year-round persistent rip channels with predominantly near-shore normal incident waves (MacMahan et al., 2005).

For a given sea state (the significant wave height and the peak wave period were about $1.4 \mathrm{~m}$ and $11.4 \mathrm{~s}$, respectively), they found $K_{a x}=2.2 \mathrm{~m}^{2} . \mathrm{s}^{-1} \pm$ $0.8 \mathrm{~m}^{2} . \mathrm{s}^{-1}$ and $K_{a y}=3.8 \mathrm{~m}^{2} \cdot \mathrm{s}^{-1} \pm 1 \mathrm{~m}^{2} . \mathrm{s}^{-1}$. The relative diffusivity was estimated as being $1.5 \mathrm{~m}^{2} . \mathrm{s}^{-1}$ and $6.5 \mathrm{~m}^{2} . \mathrm{s}^{-1}$ and between $1.2 \mathrm{~m}^{2} . \mathrm{s}^{-1}$ and $3.6 \mathrm{~m}^{2} \cdot \mathrm{s}^{-1}$ in the cross-shore and alongshore directions, respectively.

In the present work we used a $3 \mathrm{D}$ fully-coupled wave-current model to study the sensitivity of the rip system to horizontal mixing used to parameterize turbulent diffusion. We tested different diffusivity values in 
agreement with the scaling of Brown et al. (2009), extended the bidimensional (2D) work of Weir et al. (2011) to three-dimensional (3D) simulations and applied the vortex force formalism (McWilliams et al., 2004; Ardhuin et al., 2008b; Bennis et al., 2011). The main findings of our study are: a) CEW are modulated by horizontal mixing, with maximum impact at high resolution ; b) Horizontal mixing acts on the flow in a similar way to $\mathrm{CEW}$; c) CEW are negligible when using a high mixing coefficient; and, d) the convergence of the waves towards the rip channel is modulated by both horizontal mixing and spatial resolution.

The rest of this paper is organised as follows: the method used, including the governing equations and details about the simulations, is described in section 2 ; results are presented in section 3 , and we summarize our findings in section 4 .

\section{Methods}

The 3D fully-coupled MARS-WAVEWATCH III model (more details in Bennis et al., 2011, 2014) combines the MARS3D hydrodynamic model (Lazure and Dumas, 2008) and the WAVEWATCH III wave model (Tolman, 2009), hereafter WW3. Exchanges of information between the models are managed by the PALM coupler (Buis et al., 2008). We can easily investigate the feedback of currents on waves by turning off the coupling in test simulations. All the results presented here were obtained using a non stationary coupling procedure. Results obtained for cases with and without feedback are referred to hereafter as WEC+CEW and WEC-only, respectively (WEC refers to wave effects on currents and, the behaviors ascribed to CEW were obtained from the difference between WEC+CEW and WEC-only fields).

WW3 is a phase-averaged wave model. The wave action density spectrum $N$ ( $N$ being a function of time, space, wave number and direction) is solved as:

$$
\frac{D N}{D t}=\frac{Q}{\sigma}
$$

where $Q$ represents the total source and sink terms, including non-linear interactions, bottom friction, wave dissipation and bottom scattering (more details in Tolman (2009)) and $\sigma$ is the intrinsic wave radian frequency. High-order conservative numerical schemes are used for spatial discretization. A CFL (Courant-Friedrichs-Lewy) condition exists, binding the discretizations in time and in space. 
MARS3D uses the pressure projection method to solve the 3D, unsteady, Navier-Stokes equations under Boussinesq and hydrostatic assumptions. Finite difference schemes are used for the spatial discretization performed on an Arakawa-C grid (Lazure and Dumas, 2008). The model uses an ADI (Alternating Direction Implicit) time scheme (Bourchtein and Bourchtein, 2006), although, the time step of MARS3D is constrained by the CFL condition of WW3 in order to avoid too great a time shift between the two models.

Fig. 1 shows the bathymetry for the problem, given the geometry and the computational domain. Since we are interested in rip currents, the rip channels are near the center of the domain and periodic boundary conditions are used at the lateral boundaries. Neumann boundary conditions are employed at the surface and bottom, with the additional parameterization according to Soulsby (1995) for bottom friction (Bennis et al., 2014). Offshore, open boundary conditions are applied based on the method of characteristics (e.g., Zwillinger, 1989). This method consists in complementing the physical boundary conditions using characteristic pathways around which the solution is computed. The scheme of Walstra et al. (2000) is used to parameterize the modification of vertical mixing by waves near the surface and bottom.

The flow is forced by the normal propagation of an incident wave on a barred beach. Each test case uses a regular horizontal grid. The time step varies according to the size of the mesh to ensure the numerical stability of the models and the time synchronization of the coupled system. The coupling time step is also different from the time steps of the two models.

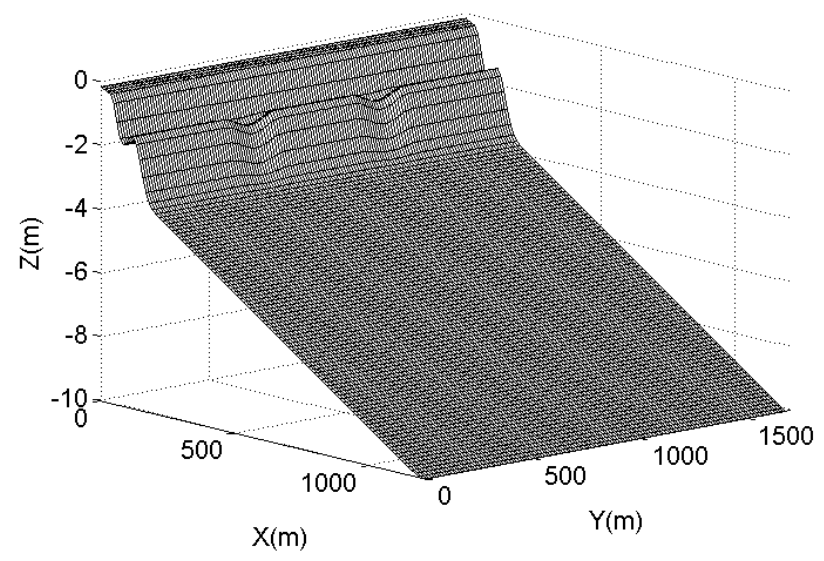

Figure 1: Bathymetry 


\subsection{Governing equations}

Wave forcing was integrated into the hydrodynamic model with the vortex force method (McWilliams et al., 2004; Ardhuin et al., 2008b). This method has been validated for surf zone cases (e.g., Kumar et al. (2012), Moghimi et al. (2012) and Bennis et al. (2014)) and gives good agreement with in-situ and laboratory data. The main advantage of the method is to consider the mean flow instead of the total momentum (Ardhuin et al., 2008b; Bennis et al., 2011), avoiding the difficult modeling of the vertical flux of momentum (Ardhuin et al., 2008a).

The equations of motion for a wave-forced, 3D, incompressible, unsteady, hydrostatic, constant-density flow are given in Ardhuin et al. (2008b) and Bennis et al. (2011). Their generic formulation is:

$$
\begin{aligned}
\frac{\mathrm{DU}}{\mathrm{Dt}} & =\mathbf{S}_{\mathbf{E P G}}+\mathbf{S}_{\mathbf{V M}}+\mathbf{S}_{\mathbf{H M}}+\mathbf{S}_{\mathbf{W P}} \\
& +\mathbf{S}_{\mathbf{B A}}+\mathbf{S}_{\mathrm{BBL}}+\mathbf{S}_{\mathbf{V F}}
\end{aligned}
$$

where $\mathcal{U}=(U, V, W)$ is the $3 \mathrm{D}$ quasi-Eulerian velocity computed as the Lagrangian velocity minus the Stokes drift. The source terms $\mathbf{S}_{\mathbf{E P G}}, \mathbf{S}_{\mathbf{V M}}$, $\mathbf{S}_{\mathbf{H M}}, \mathbf{S}_{\mathbf{B A}}, \mathbf{S}_{\mathbf{B B L}}, \mathbf{S}_{\mathbf{V F}}$ and $\mathbf{S}_{\mathbf{W P}}$ refer to the external pressure gradient, vertical mixing, horizontal mixing, breaking acceleration, streaming, vortex force and wave-induced pressure gradient, respectively. These equations are similar those of McWilliams et al. (2004) used in Uchiyama et al. (2010) and Kumar et al. (2012).

The $\mathrm{k}-\epsilon$ turbulent closure scheme, modified according to Walstra et al. (2000), is used to model vertical mixing (Bennis et al., 2014). Horizontal mixing is computed as:

$$
\begin{aligned}
& S_{H M, x}=\frac{1}{\rho}\left(\frac{\partial\left(\rho \nu_{H} \frac{\partial U}{\partial x}\right)}{\partial x}+\frac{\partial\left(\rho \nu_{H} \frac{\partial U}{\partial y}\right)}{\partial y}\right) \\
& S_{H M, y}=\frac{1}{\rho}\left(\frac{\partial\left(\rho \nu_{H} \frac{\partial V}{\partial x}\right)}{\partial x}+\frac{\partial\left(\rho \nu_{H} \frac{\partial V}{\partial y}\right)}{\partial y}\right)
\end{aligned}
$$

where $S_{H M, x}$ and $S_{H M, y}$ are the cross-shore and alongshore components of $\mathbf{S}_{\mathbf{H M}}$, respectively. $\rho$ is the water density, and $\nu_{H}$ is the turbulent kinematic viscosity also known as turbulent diffusivity. Here, $\nu_{H}$ is constant horizontally, with values between $0.2 \mathrm{~m}^{2} . \mathrm{s}^{-1}$ and $3.9 \mathrm{~m}^{2} . \mathrm{s}^{-1}$ (see below for 
more details). Kumar et al. (2012) also used $0.2 \mathrm{~m}^{2} . \mathrm{s}^{-1}$ to simulate rip currents while Yu and Slinn (2003) used a numerical diffusivity to suppress the sub-grid scale noise.

As the rip system is very sensitive to the modeling of wave breaking, we choose the same parameterization for wave breaking dissipation as Weir et al. (2011) and Yu and Slinn (2003). The well-referenced parameterizations of Thornton and Guza (1983) (hereafter TG83) and Church and Thornton (1993) (hereafter CT93) were therefore implemented in WW3 according to the following formulations:

- CT93:

$$
\mathrm{Q}_{\mathrm{CT} 93}=1.5 \sqrt{\pi} f_{p} B^{3} \frac{H_{r m s}}{h} M\left(1-\frac{1}{\left(1+\left(\frac{H_{r m s}}{H_{m}}\right)^{2}\right)^{5 / 2}}\right) F(k, \theta),
$$

where $M=1+\tanh \left(8\left(\frac{H_{r m s}}{H_{m}}-1\right)\right) \cdot H_{r m s}$ is the root-mean square significant wave height, $H_{m}$ is the maximum height that can be reached by waves without breaking (here $H_{m}=\gamma h$, where $\gamma$ is a tunable model parameter and $h$ is the water depth), $f_{p}$ is the peak wave frequency, $B$ is a tunable model parameter, $k$ is the mean wave number, $\theta$ is the mean wave direction, and $F(k, \theta)$ is the wave spectrum.

- TG83:

$$
\mathrm{Q}_{\mathrm{TG} 83}=48 \sqrt{\pi} f_{p} B^{3} \frac{\left(2 m_{0}\right)^{5 / 2}}{H_{m}^{4} h} F(k, \theta)
$$

where $m_{0}$ is the 0 -order moment of the variance density directional spectrum. To fit the data, $B$ and $\gamma$ are set to 1.52 and 0.35 , respectively.

$\mathrm{Q}_{\mathrm{CT} 93}$ or $\mathrm{Q}_{\mathrm{TG} 83}$ are included in the wave action equation computed by WW3 (see Eq. (1)) as a fraction, $\mathrm{Qbr}_{\mathrm{br}}$, of the $\mathrm{Q}$ term. $\mathrm{Q}_{\mathrm{br}}$ is equal to $\frac{\varepsilon_{b}}{\rho g \sigma}$ of $\mathrm{Yu}$ and Slinn (2003) and Weir et al. (2011), where $\epsilon_{b}$ is their dissipation function. To compute $\mathbf{S}_{\mathbf{B A}}$ in Eq. (2), $\mathrm{Q}_{\mathrm{br}}$ is integrated over all directions and wave numbers and then distributed over a characteristic depth:

$$
\mathbf{S}_{\mathbf{B A}}=\int \frac{\left(k_{x}, k_{y}\right)}{k C_{g}} Q_{b r}(k, \theta) \delta_{z} d k d \theta
$$


where $k_{x}=k \cos \theta, k_{y}=k \sin \theta$ and where $\delta_{z}$ allows the distribution of $\mathbf{S}_{\mathbf{B A}}$ over the water column. $C_{g}$ is the wave group velocity. $\mathbf{S}_{\mathbf{B A}}$ is equivalent to B in eq. (9) from Weir et al. (2011).

\section{$2.2 \quad$ Experiments}

We aimed to study the dependence of the flow response on horizontal mixing and compared the two coupling modes. In our model, the bedform is an approxi-

mation of the beach profile measured at Duck, North Carolina, on October 11, 1990 (Fig. 1). Its analytical expression, $h(x, y)$, was given by $\mathrm{Yu}$ and Slinn (2003) as:

$$
\begin{aligned}
h_{0}(x) & =\left(a_{1}-\frac{a_{1}}{\gamma_{1}}\right) \tanh \left(\frac{b_{1} x}{a_{1}}\right) \\
& +\frac{b_{1} x}{\gamma_{1}}-a_{2} \exp \left[-5\left(\frac{x-x_{c}}{x_{c}}\right)^{2}\right]
\end{aligned}
$$

where $x_{c}=80 \mathrm{~m}$ is the location of the longshore bar. $\gamma_{1}, a_{1}, b_{1}$ and $a_{2}$ are set to $11.74,2.97 \mathrm{~m}, 0.075,1.5 \mathrm{~m}$, respectively. Adding a perturbation at the longshore bar, the following bottom profile is obtained:

$$
h(x, y)=h_{0}(x)+h_{1}(x, y)
$$

with

$$
h_{1}(x, y)=h_{0}(x) \epsilon \cos \left(\frac{2 \pi y}{\lambda}\right) \exp \left[-5\left(\frac{x-x_{c}}{x_{c}}\right)^{2}\right]
$$

where $\epsilon=0.1 \mathrm{~m}$ and $\lambda=256 \mathrm{~m}$ are the magnitude and the wavelength of the perturbation, respectively. Weir et al. (2011) used a perturbation that was only very slightly different from this one.

A narrow Gaussian wave spectrum is used at the offshore boundary to simulate monochromatic waves as in Weir et al. (2011). The offshore wave characteristics and some information on bottom topography are given in Table 1.

The loss of information caused by the use of a coarse grid should be noted and need to develop more suitable parameterizations in the future. After testing resolutions of $3,6,12$ and $24 \mathrm{~m}$, we discuss here the results with 3 and 24-m of spatial resolution because they are the most representative ones for the study of the resolution effects. 


\begin{tabular}{|l|r|}
\hline Parameter & Value \\
\hline Significant wave height & $H_{s}=1.3 \mathrm{~m}$ \\
Peak wave period & $T=10 \mathrm{~s}$ \\
Mean wave direction & $\theta=90^{\circ}$ \\
Magnitude of perturbation & $\epsilon=0.1$ \\
Spacing of rip channels & $\lambda=256 \mathrm{~m}$ \\
\hline
\end{tabular}

Table 1: Common characteristics of all simulations

243 Sixteen different test cases were carried out that differed in their horizontal resolution, horizontal mixing and coupling mode (Table 2).

The coupling time step is set to $1 \mathrm{~s}$ while the model time step is $0.2 \mathrm{~s}$ and $0.5 \mathrm{~s}$ for WW3 and MARS3D, respectively. The simulated time is one hour for each simulation. We used 15 sigma levels that are evenly distributed over the vertical. Both coupling modes (WEC-only and WEC+CEW) use the same set of parameters to ensure a comparison as clean as possible. For each resolution, sensitivity tests on mixing were carried out with a variable $\nu_{H}$ (Table 2). The maximum values of $\nu_{H}$ are in the range given by Brown 2 et al. (2009). After many tests, we did not keep the values greater than $2532.0 \mathrm{~m}^{2} \cdot \mathrm{s}^{-1}$ (for the resolution of $3 \mathrm{~m}$ ) and $3.9 \mathrm{~m}^{2} . \mathrm{s}^{-1}(24 \mathrm{~m})$ because they 254 added no new information. Finally, the values of $3.9 \mathrm{~m}^{2} . \mathrm{s}^{-1}$ and $0.35 \mathrm{~m}^{2} . \mathrm{s}^{-1}$ were given by Okubo (1971) for resolutions of $24 \mathrm{~m}$ and $3 \mathrm{~m}$, respectively.

\begin{tabular}{|l|l|l|l|l|l|l|l|}
\hline \multicolumn{2}{|l|}{} & $\nu_{\mathrm{H}}=$ & $\nu_{\mathrm{H}}=$ & $\nu_{\mathrm{H}}=$ & $\nu_{\mathrm{H}}=$ & $\nu_{\mathrm{H}}=$ & $\nu_{\mathrm{H}}=$ \\
& $0.2 \mathrm{~m}^{2} / \mathrm{s}$ & $0.35 \mathrm{~m}^{2} / \mathrm{s}$ & $0.6 \mathrm{~m}^{2} / \mathrm{s}$ & $1.0 \mathrm{~m}^{2} / \mathrm{s}$ & $2.0 \mathrm{~m}^{2} / \mathrm{s}$ & $3.9 \mathrm{~m}^{2} / \mathrm{s}$ \\
\hline$\Delta_{x y}=$ & WEC-only & $\mathrm{x}$ & $\mathrm{x}$ & $\mathrm{x}$ & $\mathrm{x}$ & $\mathrm{x}$ & \\
$3 \mathrm{~m}$ & WEC+CEW & $\mathrm{x}$ & $\mathrm{x}$ & $\mathrm{x}$ & $\mathrm{x}$ & $\mathrm{x}$ & \\
\hline$\Delta_{x y}=$ & WEC-only & & $\mathrm{x}$ & & $\mathrm{x}$ & & $\mathrm{x}$ \\
$24 \mathrm{~m}$ & WEC+CEW & & $\mathrm{x}$ & & $\mathrm{x}$ & & $\mathrm{x}$ \\
\hline
\end{tabular}

Table 2: List of test cases for different spatial resolutions and horizontal mixing intensities. 


\section{Results}

As mentioned in Brown et al. (2009), for a similar wave forcing $\left(H_{s}=\right.$ $1.4 \mathrm{~m}, T_{p}=11.4 \mathrm{~s}$ and $\left.\theta=0^{\circ}\right)$, the absolute and relative diffusivites are within the ranges $\left[3.1 \mathrm{~m}^{2} . \mathrm{s}^{-1} ; 5.6 \mathrm{~m}^{2} . \mathrm{s}^{-1}\right]$ and $\left[1.9 \mathrm{~m}^{2} . \mathrm{s}^{-1} ; 7.4 \mathrm{~m}^{2} . \mathrm{s}^{-1}\right]$, respectively. We chose $\nu_{H}$ within these ranges with a maximum set at $3.9 \mathrm{~m}^{2} \cdot \mathrm{s}^{-1}$ to improve our understanding of the rip system in these realistic hydrodynamic conditions. For comparison with the former studies of Yu and Slinn (2003), Weir et al. (2011) and Kumar et al. (2012), we also used smaller diffusivities down to the stability limit of the model, with a minimum value of $0.2 \mathrm{~m}^{2} . \mathrm{s}^{-1}$. As the former studies of $\mathrm{Yu}$ and Slinn (2003) and of Weir et al. (2011) did not use a diffusion coefficient, but an artificial diffusivity not mentioned in their papers, we consider that our case with $\nu_{H}=0.2 \mathrm{~m}^{2} . \mathrm{s}^{-1}$ to be almost equivalent to their test case. So, we investigated the impact of the value of $\nu_{H}$ on wave-current interactions for two different spatial resolutions (3 and 24 meters). The high-resolution simulations use mixing coefficients between $0.2 \mathrm{~m}^{2} \cdot \mathrm{s}^{-1}$ and $2.0 \mathrm{~m}^{2} \cdot \mathrm{s}^{-1}$. The coefficients ranged from $0.35 \mathrm{~m}^{2} \cdot \mathrm{s}^{-1}$ to $3.9 \mathrm{~m}^{2} \cdot \mathrm{s}^{-1}$ at low resolution.

The waves come from offshore with a significant wave height of 1.3 meters and a peak wave period of 10 seconds. Figure 2 shows for both parameterizations (TG83 and CT93) the alongshore-averaged root mean square significant wave height $\left(\left\langle H_{r m s}\right\rangle\right)$ and the alongshore-averaged wave breaking dissipation flux $\left.\left(<\Phi_{o c}\right\rangle\right)$ as a function of the cross-shore distance. The wave shoaling is small due to the beach slope and the offshore conditions. Wave heights are higher when using the CT93 breaking parameterization, which makes the rip unstable as explained in Weir et al (2011). $\left\langle\Phi_{o c}>\right.$ shows two peaks, each of which related to a different breaking event. All the parameterizations predict that the highest peak is located approximately 150 meters from the shore. The shapes of $\left\langle H_{r m s}\right\rangle$ and $\left\langle\Phi_{o c}\right\rangle$ are similar to the ones described in Yu and Slinn (2003) and Weir et al. (2011). In the following, TG83 is used for all the simulations.

The total change in $H_{r m s}\left(\Delta H_{r m s}\right)$ ascribed to CEW is shown as a function of the cross-shore distance in Figure 3. $\Delta H_{r m s}$ is caused by wave ray bending, which is dependent on the alongshore gradient of the rip current, and also by the current velocity flux (see Weir et al., 2011, for more details). At the highest resolution, the two processes interact. From now, the 'shallow depths' term refers to depths less than $2 \mathrm{~m}$ located at a distance up to $100 \mathrm{~m}$ from the shore. Over shallow depths, $\Delta H_{r m s}$ is negative showing that the effects of the current flux of wave energy are dominant. Elsewhere, we observe positive values inside the rip channel and negative values outside it 

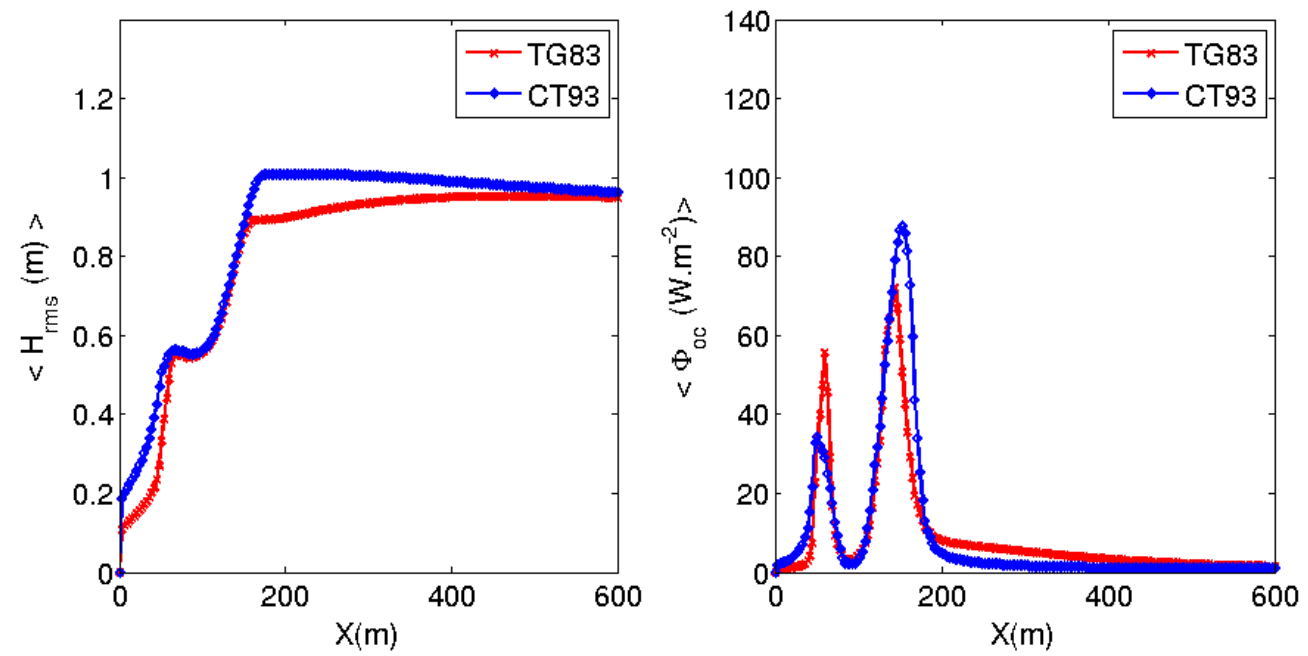

Figure 2: Alongshore-averaged root-mean square significant wave height (left panel) and alongshore-averaged wave breaking dissipation flux (right panel) computed by the TG83 and CT93 parameterizations.

(Figure 3, top row). As a result, the modulation of the wave height by $\mathrm{CEW}$ is generated by wave ray bending for $\nu_{H}=0.35 \mathrm{~m}^{2} . \mathrm{s}^{-1}$ and $\nu_{H}=2.0 \mathrm{~m}^{2} \cdot \mathrm{s}^{-1}$. For the same mixing coefficient $\left(\nu_{H}=0.35 \mathrm{~m}^{2} . \mathrm{s}^{-1}\right)$, the maximum of $\Delta H_{r m s}$ is about $0.04 \mathrm{~m}$ at high resolution while it reaches about $0.016 \mathrm{~m}$ at a spatial resolution of $24 \mathrm{~m}$. Near the shore $(\mathrm{X} \leq 100 \mathrm{~m})$, wave ray bending slightly dominates and two regions with positive values of $\Delta H_{r m s}$ appear (Figure 3 , bottom-left panel). For $\nu_{H}=3.9 \mathrm{~m}^{2} . \mathrm{s}^{-1}$, the increase in wave height inside the rip channel becomes negligible (about $0.005 \mathrm{~m}$ ). Indeed, the horizontal diffusivity is too high, leading to a large reduction in the rip velocity and its alongshore gradient. Therefore, the interactions with waves are significantly affected by mixing.

Figure 4 shows the alongshore perturbation of the wave group velocity computed as the difference between the local wave group velocity and the alonghore-averaged value. These snapshots show the simulations run at high resolution. These wave fields show that CEW cause wave divergence by refraction. For the WEC-only case, we have only refraction by bottom topography, which drives the waves towards the peak of the bar (Figure 4, top plot). When CEW are activated, refraction by currents moves the waves towards the rip channel (Figure 4, middle and bottom plots). When the smallest mixing coefficient is used $\left(\nu_{H}=0.2 \mathrm{~m}^{2} . \mathrm{s}^{-1}\right)$, refraction by 

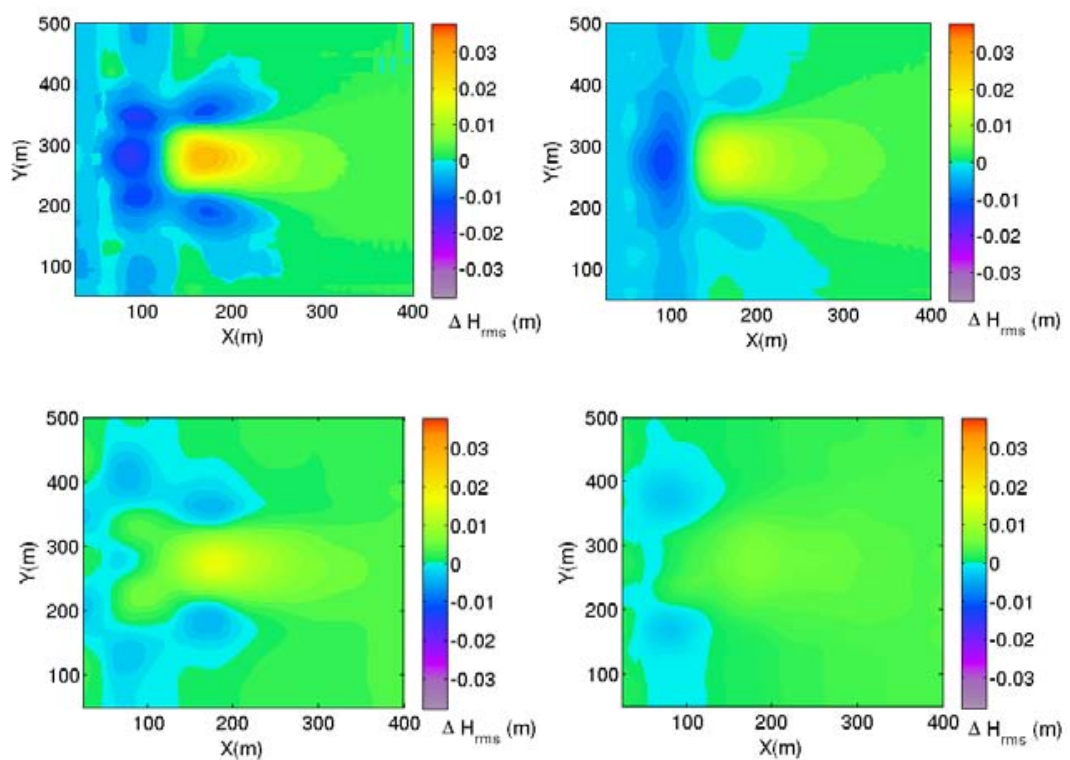

Figure 3: $\Delta H_{r m s}$ after 60 minutes. Top row: simulations with $\nu_{H}=$ $0.35 \mathrm{~m}^{2} . \mathrm{s}^{-1}$ (left panel) and with $\nu_{H}=2.0 \mathrm{~m}^{2} . \mathrm{s}^{-1}$ (right panel) at a spatial resolution of $3 \mathrm{~m}$. Bottom row: simulations with $\nu_{H}=0.35 \mathrm{~m}^{2} . \mathrm{s}^{-1}$ (left panel) and with $\nu_{H}=3.9 \mathrm{~m}^{2} \cdot \mathrm{s}^{-1}$ (right panel) at a resolution of $24 \mathrm{~m}$. Contours are equally spaced between $-0.038 \mathrm{~m}$ and $0.038 \mathrm{~m}$.

currents dominates refraction by bottom topography near the shore. Thus, waves converge towards the rip channel (Figure 4, middle plot). Horizontal mixing modifies the interactions between the two refraction processes: the higher the mixing coefficient, the weaker the rip velocity and its alongshore gradient. As a result, near the shore and with $\nu_{H}=2.0 \mathrm{~m}^{2} . \mathrm{s}^{-1}$, refraction by bottom topography dominates refraction by the rip current because the alongshore gradient of the rip current is decreased by the mixing. Thus, the effects of the rip current are not strong enough to balance the effects of the bottom topography. Finally, over shallow depths, the waves diverge from the rip channel towards the peak of the bar (Figure 4, bottom plot).

Figure 5 shows the alongshore component of the wave group velocity for $\nu_{H}=0.35 \mathrm{~m}^{2} \cdot \mathrm{s}^{-1}$ and $\nu_{H}=3.9 \mathrm{~m}^{2} \cdot \mathrm{s}^{-1}$. The results are shown for both computational grids. As explained above, refraction by bathymetry generates divergence towards the bar due to positive values of $C_{g y}$ to the right of the channel and negative values to the left (Figure 5, first column). 

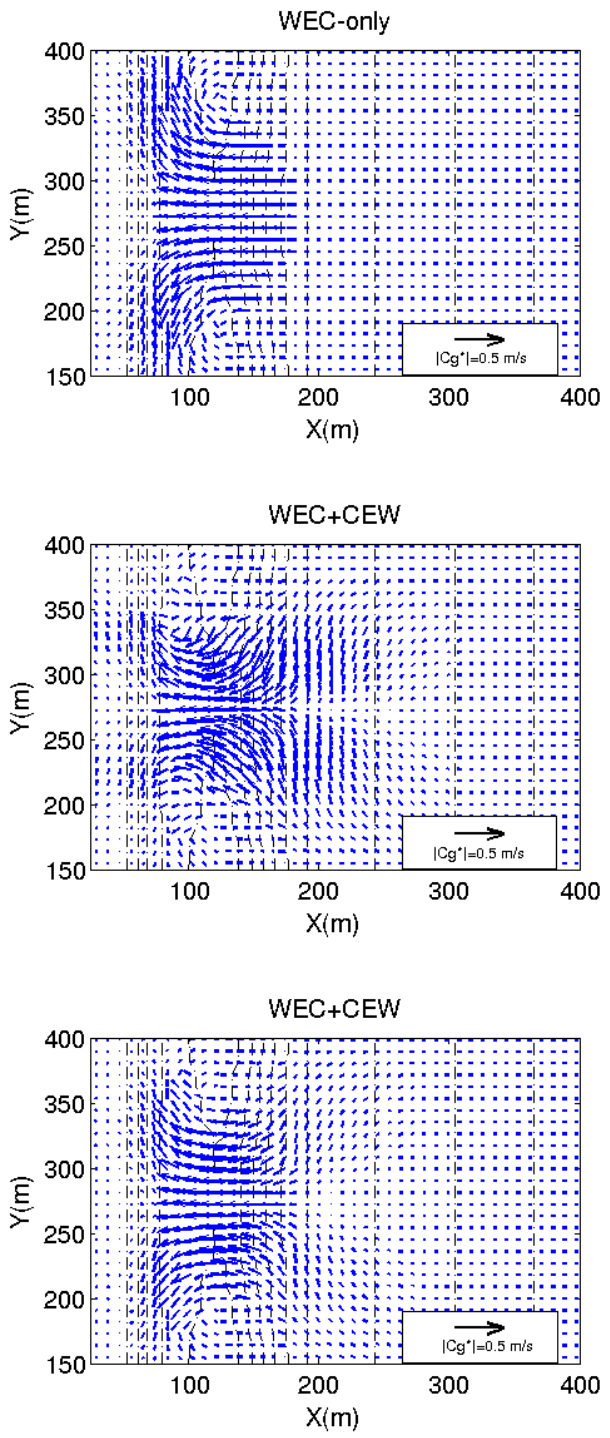

Figure 4: Alongshore perturbation of the high-resolution wave group velocity (blue arrows) at $\mathrm{t}=60$ minutes: WEC-only with $\nu_{H}=0.2 \mathrm{~m}^{2} \cdot \mathrm{s}^{-1}$ (top panel), WEC $+\mathrm{CEW}$ with $\nu_{H}=0.2 \mathrm{~m}^{2} \cdot \mathrm{s}^{-1}$ (middle panel) and WEC+CEW with $\nu_{H}=2.0 \mathrm{~m}^{2} \cdot \mathrm{s}^{-1}$ (bottom panel). Bathymetry is shown by black dashed contours. 
Refraction by currents modifies the direction, and thus produces an inverse wave motion leading to negative values of $C_{g y}$ to the right of the channel and positive values to the left (Figure 5, last column). At low resolution with $\nu_{H}=3.9 \mathrm{~m}^{2} . \mathrm{s}^{-1}$, wave diversion by the rip current is very weak and CEW are not strong enough to overcome the effects of refraction by bathymetry where the depth is shallow; therefore, the WEC+CEW alongshore group velocity looks like that of the WEC-only case (Figure 5, bottom -left and -middle plots). At the same spatial resolution with $\nu_{H}=0.35 \mathrm{~m}^{2} . \mathrm{s}^{-1}$, the patterns caused by current refraction are present for the WEC+CEW case. Indeed, as the mixing is strongly decreased from $3.9 \mathrm{~m}^{2} . \mathrm{s}^{-1}$ to $0.35 \mathrm{~m}^{2} . \mathrm{s}^{-1}$, the rip velocity and its alongshore gradient are larger and therefore interact with the waves. Near the shore $(X \leq 100 \mathrm{~m})$, we observe a special situation for which the effects of the two refraction processes cancel each other out (Figure 5, central plot): waves go straight to the shore. At the highest resolution and with the same mixing coefficient $\left(\nu_{H}=0.35 \mathrm{~m}^{2} . \mathrm{s}^{-1}\right)$, different patterns are observed for $C_{g y}$, showing the impact of spatial resolution on the wave field. Because the bathymetry and rip system are better represented on a finer grid, wave-current interactions are simulated more accurately.

Figure 6 shows the high-resolution depth-averaged vorticity fields for the WEC-only and WEC+CEW cases and for different values of $\nu_{H}\left(0.2 \mathrm{~m}^{2} \cdot \mathrm{s}^{-1}\right.$, $0.6 \mathrm{~m}^{2} . \mathrm{s}^{-1}, 1.0 \mathrm{~m}^{2} . \mathrm{s}^{-1}$ and $\left.2.0 \mathrm{~m}^{2} . \mathrm{s}^{-1}\right)$. CEW reduce the offshore extension of the flow in each case because they produce forcing effects opposed to that of the topography ( $\mathrm{Yu}$ and Slinn, 2003). Horizontal mixing, by its smoothing effect on the flow, also contributes to an attenuation of the offshore rip system. Therefore, horizontal mixing and CEW act similarly on the WEC-only vorticity field, and it is be possible to express $\mathrm{CEW}$ in terms of horizontal mixing: the WEC-only vorticity computed with $\nu_{H}=$ $2.0 \mathrm{~m}^{2} \cdot \mathrm{s}^{-1}$ is similar to the WEC+CEW vorticity obtained with $\nu_{H}=$ $1.0 \mathrm{~m}^{2} . \mathrm{s}^{-1}$ (Figure 6). When $\nu_{H}=2.0 \mathrm{~m}^{2} . \mathrm{s}^{-1}$, the WEC+CEW and WEConly fields look similar because horizontal mixing and CEW move WEC-only closer to WEC+CEW. Furthermore, we note that the WEC+CEW flow is less altered by horizontal mixing (notably with weaker offshore reduction) than the WEC-only flow. Compared with Weir et al. (2011), the evolution of the rip system at high resolution is not modified by horizontal mixing for $\nu_{H}=0.35 \mathrm{~m}^{2} \cdot \mathrm{s}^{-1}$ (Figure 7). When CEW are activated, the rip system is stable after 20 minutes (Figure 7, bottom row), but, the system continues to grow offshore for the WEC-only case. Horizontal mixing stabilizes the WEC-only flow, and thus modifies its evolution, which becomes similar to the WEC+CEW case, when the mixing coefficient is high (Figure 6, bottom row). 

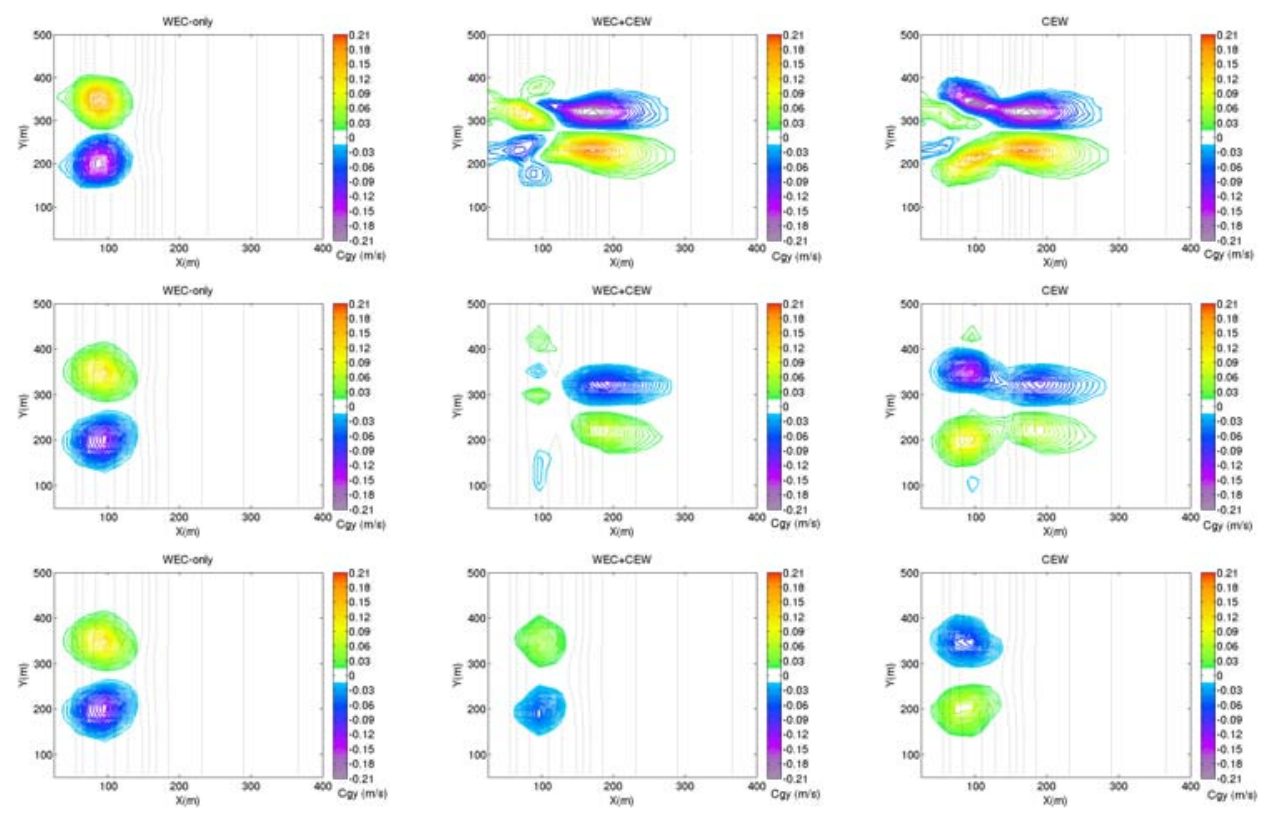

Figure 5: $C_{g_{y}}$ for cases WEC-only, WEC+CEW and CEW shown from left to right. Top row: $\Delta_{x y}=3 \mathrm{~m}$ and $\nu_{H}=0.35 \mathrm{~m}^{2} \cdot \mathrm{s}^{-1}$. Middle row: $\Delta_{x y}=$ $24 \mathrm{~m}$ and $\nu_{H}=0.35 \mathrm{~m}^{2} . \mathrm{s}^{-1}$. Bottom row: $\Delta_{x y}=24 \mathrm{~m}$ and $\nu_{H}=3.9 \mathrm{~m}^{2} . \mathrm{s}^{-1}$. All figures are plotted after 60 minutes. Contours are equally spaced from $-0.21 \mathrm{~m} . \mathrm{s}^{-1}$ to $0.21 \mathrm{~m} . \mathrm{s}^{-1}$.

Figure 8 shows the depth-averaged cross-shore and alongshore velocities normalized by the maximum velocity of the WEC-only case. Several mixing cases and both coupling modes are shown for the simulations run at $3-\mathrm{m}$ spatial resolution. We observed that the cross-shore velocity was more altered by mixing and CEW than by the alongshore velocity. As waves propagate normally to the shore, an alongshore motion is generated by refraction. For $\nu_{H}=0.2 \mathrm{~m}^{2} . \mathrm{s}^{-1}, \mathrm{CEW}$ decrease the cross-shore velocity by a factor equivalent to $50 \%$ of the maximum WEC-only velocity. The higher the mixing coefficient, the more this factor is reduced. For $\nu_{H}=2.0 \mathrm{~m}^{2} . \mathrm{s}^{-1}$, we observe a reduction by a factor equivalent to $18 \%$. As the cross-shore velocity is modified, interactions with waves are also changed. So, the increase of $H_{r m s}$ by CEW is also halved between the $0.2 \mathrm{~m}^{2} . \mathrm{s}^{-1}$ and $2.0 \mathrm{~m}^{2} . \mathrm{s}^{-1}$ cases (Figure 9). A ratio of two is maintained between the rip velocity and the wave height. The simulations run at low resolution show that even a small $\Delta H_{r m s}(0.015 \mathrm{~m}$ at its maximum) modifies the wave fields by CEW (Figure 

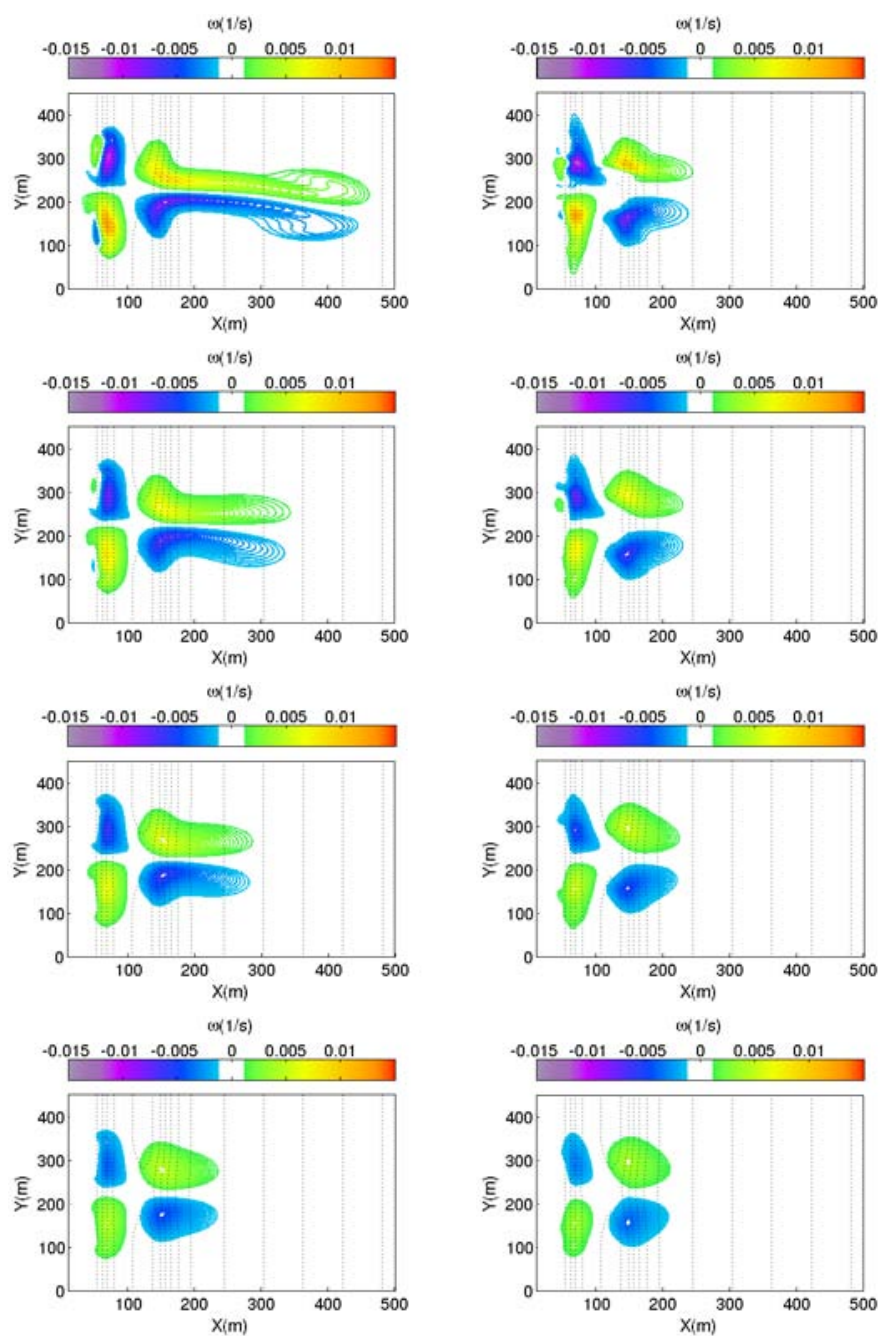

Figure 6: High resolution depth-averaged vorticity fields for the WEC-only (left column) and WEC+CEW (right column) cases after 60 minutes. Four cases of mixing are represented (from top to bottom) : $\nu_{H}=0.2 \mathrm{~m}^{2} . \mathrm{s}^{-1}$, $\nu_{H}=0.6 \mathrm{~m}^{2} . \mathrm{s}^{-1}, \nu_{H}=1.0 \mathrm{~m}^{2} . \mathrm{s}^{-1}, \nu_{H}=2.0 \mathrm{~m}^{2} . \mathrm{s}^{-1}$. Bathymetry is shown by black dotted contours.

5). In contrast, when the mixing is increased $\left(\nu_{H}=3.9 \mathrm{~m}^{2} \cdot \mathrm{s}^{-1}\right), \Delta H_{r m s}$ is about $0.005 \mathrm{~m}$ at its maximum, and the alongshore group velocity shows the same patterns for both coupling modes (Figure 5) ; the two coupling modes 
give similar results.
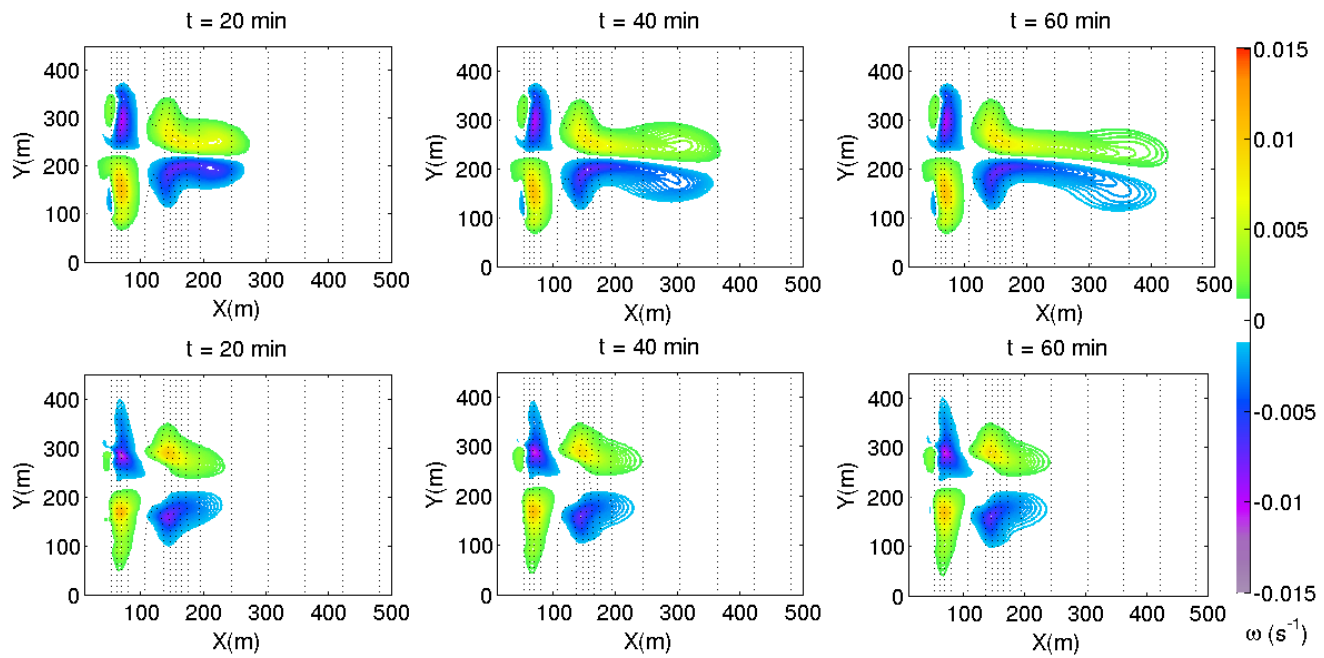

Figure 7: Evolution over time of the high-resolution depth-averaged vorticity for $\nu_{H}=0.35 \mathrm{~m}^{2} \cdot \mathrm{s}^{-1}$ : WEC-only (top row) and WEC+CEW (bottom row). Colored contours are equally spaced between $-0.015 \mathrm{~s}^{-1}$ and $0.015 \mathrm{~s}^{-1}$. Bathymetry is shown by black dotted contours.

Figure 10 shows the vertical profiles of the high-resolution 3D cross-shore velocity inside the rip channel. As in Kumar et al. (2012), the maximum rip velocity is located within the water column, and the rip current decreases towards the bottom and the surface. The vertical profiles are changed by CEW. The WEC-only velocity is more intense than the WEC+CEW velocity, particularly when the mixing coefficient is weak. For a diffusion coefficient of $0.2 \mathrm{~m}^{2} . \mathrm{s}^{-1}$, CEW reduce the maximum cross-shore velocity by $25 \%$, whereas we obtain a $8 \%$ reduction with a diffusion coefficient of $2.0 \mathrm{~m}^{2} \cdot \mathrm{s}^{-1}$. As for the barotropic fields, the offshore extension of the rip system is reduced by CEW and horizontal mixing. Mixing causes maximum alterations for the WEC-only case for which the maximum $3 \mathrm{D}$ velocity is reduced by $20 \%$ between the $0.2 \mathrm{~m}^{2} . \mathrm{s}^{-1}$ and $2.0 \mathrm{~m}^{2} . \mathrm{s}^{-1}$ cases whereas this reduction is limited to $3 \%$ when $\mathrm{CEW}$ are activated.

Horizontal mixing does not modify the vertical shear of the rip velocity because it modifies each $2 \mathrm{D}$ slice of the horizontal velocity equally in the vertical. This shows that the vertical structure is mainly forced by the set of equations used to compute the wave-current interactions, and also by the vertical mixing scheme. Wave breaking and dissipation by bottom 

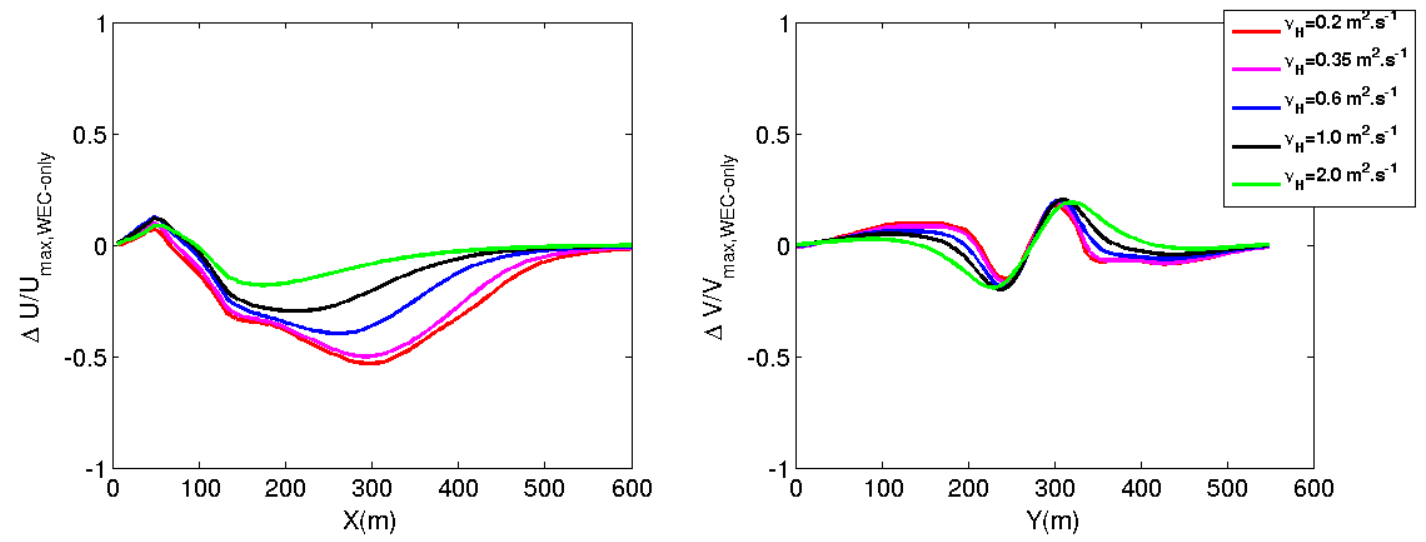

Figure 8: Total change in high-resolution depth-averaged velocities ascribed to $\mathrm{CEW}$ normalized by the maximum velocities for the WEC-only case at $t=60 \mathrm{~min}$. All mixing cases are represented. Cross-shore profiles of the cross-shore velocity inside the rip channel in $\mathrm{Y}=267 \mathrm{~m}$ (left panel) and alongshore profiles of the alongshore velocity at the velocity maximum in $\mathrm{X}=117 \mathrm{~m}$

(right panel).

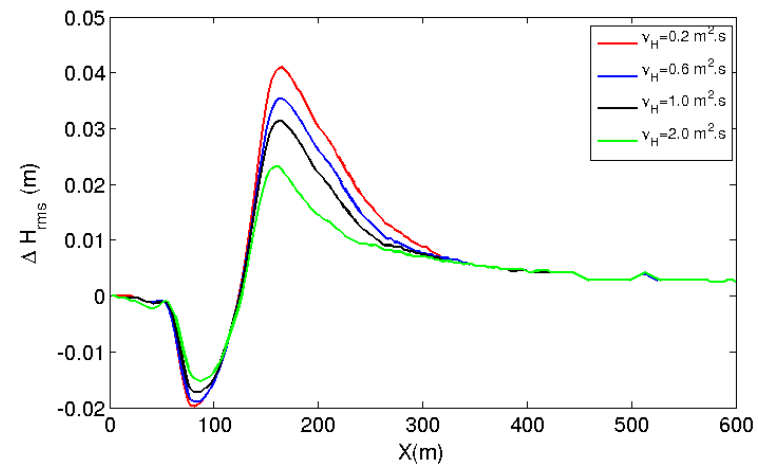

Figure 9: Total change in $\mathrm{H}_{\mathrm{rms}}$ ascribed to $\mathrm{CEW}$ inside the rip channel after 60 minutes. The simulations were run at high resolution. Four cases of mixing are shown: $\nu_{H}=0.2 \mathrm{~m}^{2} \cdot \mathrm{s}^{-1}, \nu_{H}=0.6 \mathrm{~m}^{2} \cdot \mathrm{s}^{-1}, \nu_{H}=1.0 \mathrm{~m}^{2} \cdot \mathrm{s}^{-1}$, $\nu_{H}=2.0 \mathrm{~m}^{2} \cdot \mathrm{s}^{-1}$.

friction in the wave bottom boundary layer significantly affect the vertical velocity profiles (e.g., Walstra et al., 2000 and Uchiyama et al., 2010). These processes are parameterized thanks to the vertical turbulent closure, that 


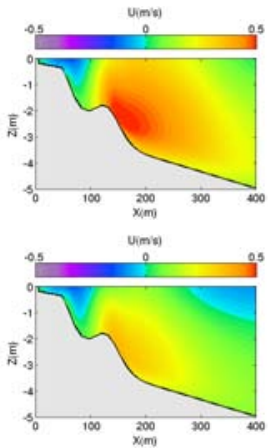

was modified to this end. The mathematical modelling of the wave-current interactions also influences the vertical profiles, as discussed in Ardhuin et al. (2008b) and Bennis et al. (2011). CEW appear too weak to change the vertical shear and act similarly to the horizontal mixing by reducing the intensity of the rip current and its offshore extension. Lastly, as reported by some previous authors (e.g., Kumar et al., 2012; Teles, 2013), the structure of the rip velocity varies with depth. Thus, 3D simulations are useful because the vertical profiles cannot be deduced from $2 \mathrm{D}$ runs. These profiles are very important when studying hydro-sedimentary motions inside the water column but also near the bottom.

Figure 10: High resolution cross-shore profiles of the 3D cross-shore velocity taken inside the rip channel for the WEC-only (top row) and WEC+CEW (bottom row) cases after 60 minutes. Four cases of mixing are represented with from left to right: $\nu_{H}=0.2 \mathrm{~m}^{2} . \mathrm{s}^{-1}, \nu_{H}=0.6 \mathrm{~m}^{2} . \mathrm{s}^{-1}, \nu_{H}=1.0 \mathrm{~m}^{2} \cdot \mathrm{s}^{-1}$, $\nu_{H}=2.0 \mathrm{~m}^{2} \cdot \mathrm{s}^{-1}$. Colored contours are equally-spaced between $-0.5 \mathrm{~m} . \mathrm{s}^{-1}$ and $0.5 \mathrm{~m} . \mathrm{s}^{-1}$.
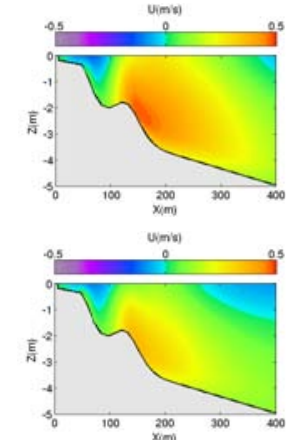
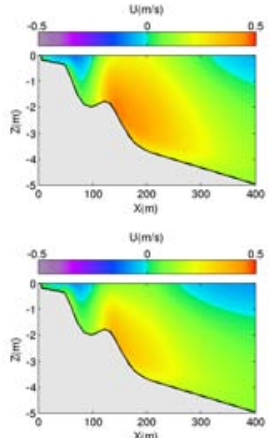
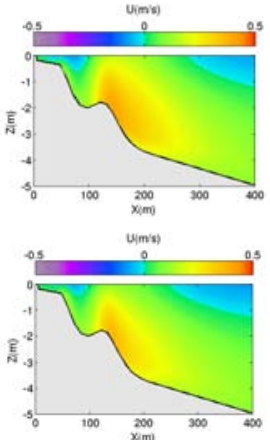

\section{Summary and conclusions}

Field measurement of diffusivity in rip current systems is difficult and many different values exist in the literature. In this paper, we numerically tested different ranges of diffusivity values in order to improve our understanding of the sensitivity of the rip system to this kind of mixing. Our test case was the same as that used by Weir et al. (2011) and Yu and Slinn (2003), and we started by revisiting their experiments. As explained above, we consider that we can reproduce their results by using the smallest diffusion coefficient $\left(0.2 \mathrm{~m}^{2} . \mathrm{s}^{-1}\right)$; a section of this paper concerns the validation of our approach. 
We then tested different diffusivity values in the range of the observations of Brown et al. (2009) for a similar wave forcing, with a maximum value of $3.9 \mathrm{~m}^{2} \cdot \mathrm{s}^{-1}$ given by Okubo (1971). For all cases, the simulations are 3D, in contrast with the previous $2 \mathrm{D}$ studies that were performed with spatial resolutions of $3-\mathrm{m}$ and $2-\mathrm{m}$. This allows us to highlight the importance of $3 \mathrm{D}$ effects. Two different spatial resolutions $(3 \mathrm{~m}$ and $24 \mathrm{~m})$ were also tested for different diffusivity values in order to understand the link between mixing, spatial resolution and wave-current interactions. For each case, we investigated how the hydrodynamic conditions and spatial resolution change the wave-current interactions in a 3D framework.

Our 2D results, obtained by integrating the horizontal momentum over depth, for a diffusion coefficient set at $0.2 \mathrm{~m}^{2} \cdot \mathrm{s}^{-1}$ and with a 3 -m spatial resolution, were tested against the results of Weir et al. (2011) and Yu and Slinn (2003). Our simulations showed a rip system quite similar to these previous studies: i) refraction by currents dominates refraction by bathymetry for shallow depths, which induces a wave motion towards the rip channel; ii) changes in wave height and wave number are produced by CEW; iii) CEW significantly reduce the offshore flow because they produce an opposite forcing to the rip motion, and thus block the system's growth, iv) CEW decrease the intensity of the flow; and, v) the flow is stabilized by $\mathrm{CEW}$. This validation shows that our $3 \mathrm{D}$ model is able to correctly reproduce the $2 \mathrm{D}$ flow simulated by pure $2 \mathrm{D}$ models. We conclude that the $3 \mathrm{D}$ effects have little impact on the depth-averaged flow although the flow shows vertical shear. This could be because the vertical shear is not strong enough to significantly impact the barotropic fields, noting that the set of equations of Bennis et al. (2011) is valid only for a weak shear. When the shear is stronger, and in these cases with the inclusion of the shear-induced pressure term of Ardhuin et al. (2008b) given in their eq. (40), the 3D effects could significantly modify the depth-averaged flow.

The horizontal resolution modifies wave-current interactions because of the coarse description of the bathymetry. For the same value of mixing coefficient $\left(0.35 \mathrm{~m}^{2} \cdot \mathrm{s}^{-1}\right)$, the change in wave height is reduced by a factor of two when the calculations are run at low resolution. This change is generated by nearshore wave ray bending and by the current velocity flux, as shown in Weir et al. (2011). At low resolution, waves go straight to the beach because wave diversion by bathymetry exactly compensates wave divergence by currents. This result suggests that the refraction by bathymetry needs to be parameterized in coarse resolution models.

When we use a mixing coefficient above $0.2 \mathrm{~m}^{2} . \mathrm{s}^{-1}$, the waves go to the peak of the bar instead of converging on the rip channel. This shows that 
refraction by bathymetry is dominant, and thus mixing changes the balance between the two refraction processes. For a diffusivity of $2.0 \mathrm{~m}^{2} . \mathrm{s}^{-1}$, the high-resolution depth-averaged vorticity without CEW is similar to the one obtained with CEW, because they have a weaker impact on the flow. We observed the same behavior at low resolution for $\nu_{H}=3.9 \mathrm{~m}^{2} \cdot \mathrm{s}^{-1}$. We noted that the high-resolution vorticity field computed without feedback and with $\nu_{H}=2.0 \mathrm{~m}^{2} . \mathrm{s}^{-1}$ looks like the one obtained for the WEC+CEW case with $\nu_{H}=1.0 \mathrm{~m}^{2} \cdot \mathrm{s}^{-1}$. We noted that mixing and CEW act in the same way: horizontal mixing reduces the offshore extension of the system, smooths the rip velocities and their alongshore gradients and stabilizes the flow. Thus, it modifies the interactions with the waves. The maximum alterations ascribed to mixing are found for the WEC-only case, showing that this flow is more sensitive than the $\mathrm{WEC}+\mathrm{CEW}$ flow. At high resolution, the maximum value of the $3 \mathrm{D}$ cross-shore velocity is reduced by $20 \%$ between the $0.2 \mathrm{~m}^{2} . \mathrm{s}^{-1}$ and $2.0 \mathrm{~m}^{2} . \mathrm{s}^{-1}$ cases, whereas this reduction is limited to $3 \%$ when CEW are activated. For the largest values of the diffusivity $\left(2 \mathrm{~m}^{2} \cdot \mathrm{s}^{-1}\right.$ and $3.9 \mathrm{~m}^{2} \cdot \mathrm{s}^{-1}$ ), the WEC-only and WEC+CEW flow patterns are similar. We hypothesize that mixing processes suppress the part of the flow that would be in disagreement with the WEC+CEW flow, indicating that the WEC-only solution for a diffusivity less than $2 \mathrm{~m}^{2} . \mathrm{s}^{-1}$ might not be realistic.

For all cases, the vertical shear of the $3 \mathrm{D}$ cross-shore velocity is not modified by CEW or horizontal mixing, which shows that it is strongly dependent on the vertical mixing scheme and on the forcing terms. In contrast, the intensity of the $3 \mathrm{D}$ velocity is strongly affected both by CEW and horizontal mixing, with similar effects. For a diffusion coefficient of $0.2 \mathrm{~m}^{2} . \mathrm{s}^{-1}$, CEW reduce the maximum 3D cross-shore velocity by $25 \%$, whereas we obtain an $8 \%$ reduction with a diffusion coefficient of $2.0 \mathrm{~m}^{2} . \mathrm{s}^{-1}$. The two mechanisms decrease the velocity and its alongshore gradient, and reduce its offshore extension.

To conclude, horizontal mixing was found to have direct impacts on wave-current interactions. We showed that the conclusions of Weir et al. (2011) and Yu and Slinn (2003) depend on both horizontal mixing and spatial resolution. When a larger mixing is used (here above $2.5 \mathrm{~m}^{2} . \mathrm{s}^{-1}$ ), CEW vanish. This result is important because the wave-current models are also used to simulate coastal seas where mixing is taken into account to represent subgrid scale processes. In the future, these results could be applied to 3D morphodynamic studies. 


\section{Acknowledgments}

The authors thank B. Weir for his expert advice and F. Ardhuin for his help and the useful comments. The authors thank three anonymous reviewers for their input. A.-C. B. is supported by the Université de Caen Normandie. A.-C. B. also acknowledges the support of a post-doctoral grant from Université de Bretagne Occidentale, and the PREVIMER project. A.-C. B. was also supported by a FP7-ERC grant number 240009 for the IOWAGA project. F.D. is supported by Ifremer and the PREVIMER project. B.B. is supported by CNRS.

\section{References}

Ardhuin, F., A. D. Jenkins, and K. Belibassakis, 2008a: Commentary on 'the three-dimensional current and surface wave equations' by George Mellor. J. Phys. Oceanogr., 38, 1340-1349.

Ardhuin, F., N. Rascle, and K. A. Belibassakis, 2008b: Explicit wave-averaged primitive equations using a generalized Lagrangian mean. Ocean Modelling, 20, 35-60, doi:10.1016/j.ocemod.2007.07.001.

Battjes, J. A., 1975: Modelling of turbulence in surf zone. Symposium on Modelling Techniques, San Francisco, ASCE, 1050-1061.

Bennis, A.-C., F. Ardhuin, and F. Dumas, 2011: On the coupling of wave and three-dimensional circulation models: Choice of theoretical framework, practical implementation and adiabatic tests. Ocean Modelling, 40, 260-272.

Bennis, A.-C., F. Dumas, F. Ardhuin, and B. Blanke, 2014: Mixing parameterization: impacts on rip currents and wave set-up. Ocean Engineering, 42, 213-227.

Bourchtein, A. and L. Bourchtein, 2006: Modified time splitting scheme for shallow water equations. Mathematics and Computers in Simulation, 73, $52-64$.

Brown, J., J. MacMahan, A. Reiners, and E. Thornton, 2009: Surf zone diffusivity on a rip-channeled beach. J. Geophys. Res., 114, C11 015.

Buis, S., A. Piacentini, and D. Déclat, 2008: PALM: A computational framework for assembling high performance computing applications. Concurrency Computat.: Pract. Exper., 18 (2), 247-262. 
Church, J. C. and E. B. Thornton, 1993: Effects of breaking wave induced turbulence within a longshore current model. Coastal Eng., 20, 1-28.

Clark, D. B., S. Elgar, and B. Raubenheimer, 2012: Vorticity generation by short-crested wave breaking. Geophys. Res. Lett., 39, L24604.

Clarke, L., D. Ackerman, and J. Largier, 2007: Dye dispersion in the surfzone: Measurements and simple models. Continental Shelf Research, 27, 650-669.

Falques, A., A. Montoto, and D. Vila, 1999: A note on hydrodynamic instabilities and horizontal circulation in the surf zone. J. Geophys. Res., 104 (C9), 20 605-20615.

Fortunato, A. and A. Baptista, 1994: Localized sigma coordinates for the vertical structure of hydrodynamic models. Proceedings of the Third International Conference on Estuarine and Coastal Modeling, New York.

Grant, S., J. H. Kim, B. H. Jones, S. A. Jenkins, and J. Wasyl, 2005: Surf zone entrainment along-shore transport and human health implications of pollution from tidal outlets. J. Geophys. Res., 110, C10 025.

Haas, K. A., I. A. Svendsen, and M. C. Haller, 1998: Numerical modeling of nearshore circulations on a barred beach with rip channels, paper presented at the 26th conference on coastal engineering. Am. Soc. of Civ. Eng.

Huang, W. and M. Spaulding, 1996: Modelling horizontal diffusion with sigma coordinate system. Journal of Hydraulic Engineering, 122, 349-352.

Huang, W. and M. Spaulding, 2002: Reducing horizontal diffusion errors in $\sigma$-coordinate coastal ocean models with a second-order lagrangian-interpolation finite-difference scheme. Ocean Engineering, 29, 495-512.

Imann, D. L., R. J. Trait, and C. E. Nordstrom, 1971: Mixing in the surfzone. J. Geophys. Res., 76, 3493-3514.

Johnson, D. and C. Pattiaratchi, 2006: Boussinesq modelling of transient rip currents. Coastal Engineering, 53, 419-439.

Kumar, N., G. Voulgaris, J. C. Warner, and M. Olabarrieta, 2012: Implementation of the vortex force formalism in the coupled ocean-atmosphere-wave-sediment transport (COAWST) modeling system for inner shelf and surf zone applications. Ocean Modelling, 47, 65-95. 
Lazure, P. and F. Dumas, 2008: An external-internal mode coupling for a $3 \mathrm{~d}$ hydrodynamical model for applications at regional scale (MARS). Adv. Water Resources, 31, 233-250.

Leblond, P. H. and C. L. Tang, 1974: On energy coupling between waves and rip currents. J. Geophys. Res., 79, 811-816.

Long, J. W. and H. T. Ozkan-Haller, 2005: Offshore controls on nearshore rip currents. J. Geophys. Res., 110, C12 007, doi:10.1029/2005JC003018.

MacMahan, J. H., E. B. Thornton, and A. J. Reniers, 2006: Rip current review. Coastal Eng., 53, 191-208.

MacMahan, J. H., E. B. Thornton, T. P. Stanton, and A. J. H. M. Reniers, 2005: RIPEX-Rip currents on a shore-connected shoal beach. Marine Geology, 218, 113-134.

McWilliams, J. C., J. M. Restrepo, and E. M. Lane, 2004: An asymptotic theory for the interaction of waves and currents in coastal waters. J. Fluid Mech., 511, 135-178.

Mellor, G. L. and A. F. Blumberg, 1985: Modelling vertical and horizontal diffusivities with the sigma coordinate system. Monthly Weather Review, 113, 1379-1383.

Moghimi, S., K. Klingbeil, U. Grawe, and H. Burchard, 2012: A direct comparison of the depth-dependent radiation stress method and a vortex force formulation within a three-dimensional ocean model. Ocean Modelling, 1-38.

Okubo, A., 1971: Oceanic diffusion diagram. Deep Sea Research, 18, 789-802.

Sagaut, P., S. Deck, and M. Terracol, 2013: Multiscale and multiresolution approaches in turbulence, 2nd edition. Imperial College Press, 450 pp.

Shepard, F. P., 1936: Undertow, rip tide or rip current. Science, 84, 181-182.

Shepard, F. P., K. O. Emery, and E. C. L. Fond, 1941: Rip currents: A process of geological importance. Journal of Geology, 49, 337-369.

Smagorinsky, J., 1963: General circulations experiments with the primitive equations i. the basic experiment. Monthly Weather Review, 8, 99-165. 
Soulsby, R. L., 1995: Bed shear stresses due to combined waves and currents. In: Stive, M., Fredsøe, J., Hamm, L., Soulsby, R., Teisson, C., Winterwerp, J. (Eds). Advances in Coastal Morphodynamics, Delft Hydraulics, Delft, The Netherlands, 420-423.

Spydell, M. and F. Feddersen, 2009: Lagrangian drifter dispersion in the surf zone: Directionally spread, normally incident waves. J. Phys. Oceanogr., 39, 809-830.

Teles, M. J., 2013: Wave-current modelling at local and regional scales. Ph.D. thesis, Instituto Superior Tecnico, Lisbon, Portugal.

Thornton, E. B. and R. T. Guza, 1983: Transformation of wave height distribution. J. Geophys. Res., 88 (C10), 5,925-5,938.

Tolman, H. L., 2009: User manual and system documentation of WAVEWATCH-III ${ }^{\mathrm{TM}}$ version 3.14. Tech. Rep. 276, NOAA/NWS/NCEP/MMAB.

Uchiyama, Y., J. C. McWilliams, and A. F. Shchepetkin, 2010: Wave-current interaction in oceanic circulation model with a vortex-force formalism Application to the surf zone. Ocean Modelling, 34, 16-35.

Walstra, D. J. R., J. Roelvink, and J. Groeneweg, 2000: Calculation of wave-driven currents in a 3D mean flow model. Proceedings of the 27th international conference on coastal engineering, Sydney, ASCE, Vol. 2, 1050-1063.

Weir, B., Y. Uchiyama, E. M. Lane, J. M. Restrepo, and J. C. McWilliams, 2011: A vortex force analysis of the interaction of rip currents and surface gravity waves. J. Geophys. Res., 116, C05 001.

Yu, J., 2006: On the instability leading to rip currents due to wave-current interaction. J. Fluid Mech., 549, 403-428.

Yu, J. and D. N. Slinn, 2003: Effects of wave-current interaction on rip currents. J. Geophys. Res., 108 (C3), 3088.

Zwillinger, D., 1989: Handbook of differential equations. Academic Press, 1-665 pp. 